\title{
Educação em saúde com prostitutas de Fortaleza: relato de experiência
}

\author{
Health education with prostitutes of Fortaleza: case report
}

\section{Educación en salud con las prostitutes de Fortaleza: relato de vivencia}

\author{
Maria Leonor Costa de Moraes', Priscila Bomfim Costa", \\ Priscila de Souza Aquino'I", Ana Karina Bezerra Pinheiro'v
}

\section{RESUMO}

As prostitutas, mesmo estando informadas sobre as DST nos seus aspectos clínicos, sentem dificuldade na manutenção do uso do preservativo. Percebe-se que o fator econômico é sentido por elas de forma mais concreta do que a necessidade de cuidar de sua saúde. Assim, realizou-se este estudo com o objetivo de relatar a experiência de educação em saúde com prostitutas atuantes em Fortaleza no ambiente da sala de espera da consulta de Enfermagem em Ginecologia no ano de 2006. Participaram das oficinas 55 mulheres, distribuídas em seis dias. As oficinas foram divididas em cinco momentos: auto-valorização; exame ginecológico; auto-estima; concorrência, competição e autoconfiança; direitos das mulheres e violência contra a mulher. Verificouse que o trabalho de educação em saúde em grupo de prostitutas viabiliza sentimento de inclusão social, eqüidade e auto-valorização, pois as participantes se identificaram entre si, refletindo a sua circunstância pessoal na condição de trabalho, vida e saúde. Fortaleceram as relações humanas e transformaram alguns conteúdos abordados em aprendizado.

Palavras chave: Educação em saúde; Prostituição; Enfermagem.

\section{ABSTRACT}

The prostitutes, even being aware of STD and its clinical aspects, find difficult using preservatives. It was observed that financial problems are more appealing to them than taking care of their own health. Therefore, this study shows the experience of providing health education to active prostitutes in Fortaleza. The wait room from the office of Nursing in Gynecology in 2006 was the local of the interviews. During six days, 55 women attended to the workshops. The workshops consisted of five moments: building up your self worth; gynecological examination; competing, competition and self-belief; self-esteem; Women's Rights and violence against women. Likewise, it was verified that health education to groups of prostitutes can provide them feeling of social inclusion, fairness and building up their self worth, once the participants recognized themselves in the group. The human relationships were fortified and some of the topics presented were really learned.

Key words: Health education; Prostitution; Nursing.

\section{RESUMEN}

Las prostitutas, mismo siendo informadas sobre las DST en sus aspectos clínicos, sienten dificultad en el mantenimiento del uso del condón. Percibimos que el factor económico es sentido por ellas de una forma más concreta que la necesidad del cuidado de su salud. Así, este estudio fue hecho con el objetivo de decir la vivencia de educación en salud con prostitutas con actuación en Fortaleza en el ambiente de la sala espera de la consulta de Enfermería en Ginecología en el año de 2006. Participaron de los talleres 55 mujeres, distribuidas en seis días. Los talleres fueron divididos en cinco momentos: autovaluación; examen ginecológico; autoestima; concurrencia, competición y autoconfianza; los derechos de las mujeres y violencia contra la mujer. Verificase que el trabajo de educación en salud

\footnotetext{
Acadêmica de Enfermagem do $5^{\circ}$ semestre da Universidade Federal do Ceará. Bolsista do Programa de Educação Tutorial (PET-Enfermagem-UFC). E-mail: leonor morais2004@yahoo.com.br.

Acadêmica de Enfermagem do 6o semestre da Universidade Federal do Ceará. Bolsista do Programa de Educação Tutorial (PET-Enfermagem-UFC). E-mail: priscilinhapbc@yahoo.com.br

Enfermeira. Mestranda em Enfermagem pela Universidade Federal do Ceará. Bolsista CAPES. E-mail: priscilapetenf@yahoo.com.br.

iv Enfermeira. Doutora em Enfermagem. Professora Adjunto II da Universidade Federal do Ceará. E-mail: anakarina.pinheiro@bol.com.br.
} 
en grupo de prostitutas originó la sensación de la inclusión social, imparcialidad y autovaluación, pues las participantes si identificaron entre sí mismo, reflejando su circunstancia personal en la condición del trabajo, vida y salud. Fortalecieran las

\section{NTRODUÇÃO}

A prostituição é reconhecida como um dos mais remotos produtos de comércio da humanidade, sendo sua história tão antiga quanto à história desta, na qual nenhuma civilização escapou à sua convivência.

A prostituição consiste em uma relação sexual entre pessoas na qual o vínculo determinante não é o afeto ou o desejo recíproco, mas sim o ato de proporcionar prazer sexual em troca de dinheiro ou outro tipo qualquer de benefício(1).

$\mathrm{Na}$ antiguidade, a prostituição era praticada por meninas como uma espécie de ritual de iniciação quando atingiam a puberdade. Em algumas civilizações, as prostitutas recebiam honras e presentes em troca de favores sexuais.

Existem fatores socioeconômicos e psicológicos que são determinantes na prostituição. A migração para os centros urbanos, a falta de emprego, condições de vida subumanas, a baixa escolaridade e a falta de perspectiva incluem-se nos primeiros, enquanto as carências afetivas, os traumas e a falta de apoio familiar são aspectos psicológicos influenciantes na inserção ao comércio sexual(2).

Atualmente, com o aumento das Doenças Sexualmente Transmissíveis e da Aids (DST/Aids), o foco das intervenções estatais passou a contemplar todos os indivíduos considerados vulneráveis, não mais os grupos de risco, alvos das ações no início da epidemia, que incluía prostitutas, homo e bissexuais ${ }^{(3)}$.

Apesar das tentativas dos órgãos de saúde pública em todo o mundo na prevenção a estas doenças, em regiões mais pobres do planeta, miséria e prostituição são palavras praticamente sinônimas ${ }^{(4)}$.

A prostituição é identificada na sociedade brasileira desde o século $X I X^{(5)}$. Além disso, o número de prostitutas tem aumentado significativamente na última década, estando a relaciones humanas y transformaran algún contenido abordado en aprendizaje.

Palabras clave: Educación en salud; Prostituición; Enfermeria.

cidade de Fortaleza entre os quatro centros do tráfico de mulheres no Brasil ${ }^{(6)}$.

Pela multiplicidade de parceiros e pela vulnerabilidade a que estão submetidas, as prostitutas podem estar sujeitas a outros problemas como más condições de vida, negociação do preservativo e até ausência deste, violência física e psicológica e baixa autoestima que interferem na qualidade de vida das mesmas.

Ainda hoje, a figura da prostituta está vinculada à transmissão das DST, devido ao comportamento sexual adotado, dito como pervertido. Porém, essa imagem é fruto do desvio do modelo padrão comportamental ${ }^{(7)}$. Em se tratando de prostitutas, tais questões contribuem para que essas mulheres tornem-se suscetíveis a crises de identidade, baixa autoestima e depressão.

Em estudo realizado em Porto Alegre sobre sintomas depressivos em uma amostra de prostitutas, foi verificada uma alta taxa de prevalência de sintomas depressivos (67\%), na qual $47,4 \%$ das entrevistadas apresentaram sintomatologia com níveis moderado a grave. Além disto, análises mostraram associação entre a presença de sintomas depressivos e uso de álcool, história de doenças sexualmente transmissíveis e falta de algum tipo de prática religiosa $^{(8)}$.

Ressalta-se, na prostituição, a desarticulação da atuação dos mecanismos de controle social formal e informal junto a essa categoria de pessoas. A Igreja, a escola e a família deveriam representar redes sociais de apoio a crianças e adolescentes em situações de risco social, submetidas à violência e à violação dos direitos humanos. Constata-se que esses jovens são mais facilmente atraídos para a rede de prostituição(4).

Segundo estudo realizado no Rio de Janeiro sobre representações sociais de profissionais de saúde sobre violência sexual 
contra a mulher, o conjunto das falas refere sofrimento, distúrbio do comportamento e relação sexual forçada. Sabe-se hoje que as mulheres atingidas ficam mais vulneráveis a outros tipos de violência, à prostituição, ao uso de drogas, à gravidez indesejada, às doenças ginecológicas, aos distúrbios sexuais, à depressão, ao suicídio e às infecções sexualmente transmissíveis ${ }^{(9)}$.

O Sistema Único de Saúde (SUS) garante que as pessoas devem ser assistidas por meio de ações de promoção, proteção e recuperação da saúde, em conjunto a ações assistenciais e preventivas $^{(10)}$.

Reconhecendo, pois, a dificuldade enfrentada pelas prostitutas em participar dos benefícios que a Saúde Pública oferece, seja por motivo de vergonha, preconceito ou depreciação da auto-estima, desenvolveu-se uma estratégia de educação em saúde com prostitutas cadastradas na Associação de Prostitutas do Ceará (APROCE) que aguardavam atendimento ginecológico.

O encontro dialógico caracteriza-se pela simplicidade e espontaneidade, não se convertendo, a primeira em simplismo, a segunda, em espontaneísmo. Dialogar não é um perguntar a esmo, um perguntar por perguntar, um responder por responder, um contentar-se por tocar a periferia, apenas, do objeto de nossa curiosidade ${ }^{(11)}$.

Nesse contexto, as prostitutas puderam agir de forma espontânea, refletindo sobre suas práticas, a importância da consulta ginecológica e da auto-valorização, despertando para mudanças de comportamentos. O desafio está em buscar um momento e um espaço para educar. Diante disso, a sala de espera se mostra como um local ideal para passar informações relevantes ao paciente, antes da consulta, e para o compartilhamento de informações com pessoas que possuam uma necessidade de saúde em comum ${ }^{(12)}$.

O enfermeiro como agente promotor de mudanças, responsável pela melhoria da situação de saúde da população, deve tornar a unidade de saúde um ambiente de interação com a comunidade, aproveitando os momentos pré-consulta (sala de espera) para o processo de orientação e reflexão. $O$ trabalho com grupos tem aumentado sua importância, sendo utilizada em diversas práticas como um grupo operativo, de auto-ajuda, grupo educativo e grupo com pessoas com problemas crônicos de saúde ${ }^{(12)}$.

Diante do exposto, realizamos o presente estudo com o objetivo de relatar a experiência de educação em saúde com prostitutas atuantes em Fortaleza no ambiente da sala de espera da consulta de Enfermagem em Ginecologia.

\section{A TRAJETÓRIA PERCORRIDA E O CONTEXTO DA EXPERI ÊNCIA}

O público-alvo da experiência foi composto por prostitutas cadastradas na APROCE, que conta com cerca de 3.500 mulheres cadastradas em todo o Ceará.

A APROCE surgiu em 1990, com o intuito de lutar por uma melhor qualidade de vida, bem como desenvolver trabalhos contra a exploração sexual infantil. A Associação promove diversas atividades, como: oficinas, projetos, palestras, seminários, cursos, produção de material informativo, distribuição de preservativos e trabalho de educação em saúde, além de fiscalizar e denunciar os locais de prostituição infanto-juvenil.

Participaram das oficinas 55 mulheres, distribuídas em dias distintos, totalizando um máximo de oito mulheres por encontro. As consultas foram marcadas na ocorrência da realização de uma campanha de incentivo ao exame de prevenção ginecológica, promovida pelo PET-Enfermagem-UFC em parceria com a APROCE, em três zonas selecionadas de Fortaleza: o Centro, o Farol do Mucuripe e a Barra do Ceará, com o objetivo de sensibilizar essas mulheres a se prevenirem contra o câncer de colo de útero.

O câncer de colo do útero é a terceira neoplasia maligna mais comum e a quarta causa de morte por câncer em mulheres. Os principais fatores de risco associados ao câncer de colo uterino, são: baixa condição sócioeconômica, início precoce da atividade sexual, multiplicidade de parceiros sexuais, tabagismo, higiene íntima inadequada e uso prolongado de contraceptivos orais, fatores de risco que, na maioria, são encontrados entre as prostitutas ${ }^{(13)}$. 
O desenvolvimento das oficinas se deu nos meses de outubro e novembro de 2006, no Centro de Parto Natural (CPN) Lígia Barros Costa, órgão da Universidade Federal do Ceará (UFC) que localiza-se no bairro Planalto Pici, em Fortaleza. O CPN propõe-se a prestar um serviço de qualidade acerca do planejamento familiar, da atenção ginecológica e obstétrica.

As oficinas foram divididas em cinco momentos, abordando, respectivamente, os seguintes temas: auto-valorização; exame ginecológico; auto-estima; concorrência, competição e autoconfiança; direitos das mulheres e violência contra a mulher.

$\mathrm{O}$ estudo foi realizado com consentimento da coordenadora da unidade e o projeto foi apreciado pelo Comitê de Ética em Pesquisa da UFC e a autorização foi emitida por meio do protocolo número 289. As participantes da oficina consentiram que a exploração da experiência fosse utilizada para um relato científico.

\section{CONTRI BUI ÇÕES DA EXPERI ÊNCI A}

A metodologia de educação em saúde pode ser melhor conduzida no trabalho com grupos, pois este possibilita um enriquecimento das discussões e uma expansão de conhecimentos, viabilizando sentimentos de inclusão, eqüidade e valorização, pois os participantes se identificam entre si e desenvolvem um fortalecimento entre as relações humanas que, posteriormente, se transformam em aprendizado ${ }^{(14)}$.

O local da sala de espera ou pré-consulta é o ambiente onde os clientes aguardam o atendimento dos profissionais de saúde. É um espaço dinâmico e funcional, no qual surgem diversas subjetivações devido à interação e à troca de experiências entre pessoas distintas. Além disso, proporciona um intercâmbio entre os saberes populares e a ciência dos profissionais de saúde, não permitindo a interferência de preconceitos e convencionalismos em suas ações profissionais $^{(15)}$.

A utilização de oficinas apresenta-se como estratégia de crescimento e reflexão, na qual os participantes são estimulados a elaborar um conceito sobre si e a defender suas opiniões perante o grupo, expressando, assim, suas potencialidades, além de promover o lúdico e possibilitar a descontração e a criação de elos entre os participantes do grupo e os facilitadores das oficinas ${ }^{(16)}$.

No primeiro momento, foi realizada uma sessão quebra-gelo, na qual as participantes confeccionaram seus crachás e expuseram o nome que gostariam de ser chamadas, apresentando-se verbalmente em seguida. Nesta etapa, buscou-se estimular a desinibição e a interação das participantes, bem como a criação de um vínculo entre as mesmas e os facilitadores da oficina. Durante a confecção dos crachás, observou-se o processo de descontração e introspecção em busca da criatividade de cada uma ao decorar seu crachá.

Em estudo realizado através de um comparativo entre as técnicas utilizadas para o desenvolvimento de oficinas, verificou-se que o momento inicial de descontração ou aquecimento é fundamental para o entrosamento do grupo e para dar continuidade às fases seguintes da oficina(16).

Percebeu-se um despertar para a autovalorização, por meio do simples fato de se apresentarem perante o grupo a que estão inseridas, bem como de se auto-afirmarem por meio da escrita do nome pelo qual elas gostam de ser chamadas.

No segundo momento, foi explicada a importância do exame ginecológico para a prevenção do câncer e para o diagnóstico das DST, sendo ainda demonstrado como é realizado o exame, proporcionando às participantes a oportunidade de conhecer o instrumental utilizado na consulta e esclarecer suas dúvidas.

A realização freqüente do exame ginecológico é relevante no que diz respeito à prevenção, detecção precoce e conseqüente redução de casos de câncer de colo do útero, bem como no controle de doenças sexualmente transmissíveis, no planejamento familiar, no pré-natal e em patologias obstétricas.

Para o ano de 2006 foi estimada a incidência 19.260 novos casos de câncer do colo do útero em todo o Brasil e de 780 novos casos no Ceará(13). O câncer de colo uterino é detectado com facilidade e possui um período 
duradouro de evolução, podendo levar anos para desenvolver-se até ser diagnosticado. Essa patologia é curável, desde que seja identificada num estágio inicial e tratada de forma correta.

Diante do instrumental utilizado para a descrição das etapas do exame ginecológico, as participantes demonstraram imensa curiosidade. Observou-se um grande número de indagações e demonstrações de medo em relação ao exame, o que evidencia a falta de conhecimento sobre o assunto.

A insuficiência de informação na educação sexual das mulheres não decorre dos dias atuais. As marcas da repressão sexual transcendida de geração a geração e dependente de fatores sociais, políticos, culturais, históricos, educacionais e religiosos, tem influenciado significativamente nos modos e na conduta das pessoas, privando-as de ter acesso a conhecimento e informação, corroborando para uma saúde potencialmente mais frágil. Devido a isso, os tabus, os valores, os padrões morais e as regras ainda permeiam o sexo e a sexualidade, impedindo que estes sejam vivenciados com naturalidade e aceitos como necessidades humanas básicas ${ }^{(17)}$.

Esse estigma da repressão sexual inviabiliza até mesmo uma discussão aberta acerca da sexualidade dentre os indivíduos. Deste modo, sendo o enfermeiro responsável pela promoção da saúde e prevenção de doenças, faz-se necessário que o mesmo promova a seus clientes orientações, discussões e informações sobre sexo, sexualidade e suas implicações ${ }^{(18)}$.

Após o esclarecimento das dúvidas percebemos uma maior aceitação do exame ginecológico, o que poderá favorecer a qualidade de vida dessas mulheres.

No terceiro momento, foi realizada uma dinâmica na qual era solicitado que cada participante escrevesse em uma folha sua maior qualidade, seu maior defeito, a parte do corpo que era mais admirada e a menos admirada.

Inicialmente, no processo de busca por seus defeitos e qualidades, pôde-se constatar, na maioria das participantes, uma facilidade em listar seus defeitos e suas fraquezas, e uma dificuldade em mencionar suas qualidades, retratando assim sua baixa auto-estima e a criticidade quanto ao próprio estilo de vida e visão de si mesmas, principalmente relacionada ao forte preconceito social e pessoal.

Isto pôde ser confirmado em estudo sobre a representação social da prostituição que demonstrou que o senso comum na sociedade caracteriza as prostitutas em seres transgressores e esta imagem é absorvida pela própria prostituta ${ }^{(19)}$.

Durante as discussões, refletiu-se que qualidades e defeitos são atributos comuns a todas as pessoas, desmistificando que ser prostituta seria uma atividade desenvolvida por mulheres desqualificadas.

No quarto momento, foi entregue para cada participante um balão, um palito de dente e uma folha contendo uma figura ilustrativa de uma mulher. Foi solicitado para que cada uma pintasse a sua figura e escrevesse no verso da mesma planos, sonhos e metas que gostariam de alcançar em suas vidas. Após, foi solicitado para que cada participante dobrasse a sua figura, colocasse dentro do balão, inflasse e amarrasse o balão. $O$ facilitador pediu para que cada uma defendesse o seu sonho. A maioria das mulheres tentou destruir os sonhos das outras, representados, no caso, pelos balões. Sabe-se que a prostituição está imersa em um universo repleto de concorrência e competição.

Há concorrência neste universo e está relacionada especialmente a três aspectos principais: à restrita condição financeira dos clientes, que obriga as prostitutas a reduzir o preço do programa e a aumentar o número de programas realizados; à preferência de alguns homens por mulheres mais jovens, o que diminui o número de clientes, o preço do programa e aumenta a competição entre as mulheres de mais idade; e quando os homens procuram por prostitutas de maior experiência profissional, acarretando em concorrência acirrada entre as mulheres mais jovens, o que atenua o preço do programa ou até mesmo facilita a não utilização do preservativo, interferindo na saúde das mesmas ${ }^{(19-20)}$.

Após a atividade, refletiu-se, então, que não é necessário destruir os sonhos de outras pessoas para defender os seus, basta lutar por eles. Percebeu-se, também, nos relatos das participantes, um sentimento de autoconfiança, 
em que estas descobriram que são capazes de melhorar suas condições de vidas, pois são potentes agentes de mudança.

No quinto momento, foi realizada uma exposição de um cartaz contendo os principais direitos das mulheres. Nessa fase buscou-se falar dos direitos e discutir acerca da concretização dos mesmos, pois sendo um grupo excluído ou que se exclue, as prostitutas, muitas vezes, não concretizam a sua cidadania, por não conhecimento dos seus direitos ou por discriminação social.

Através da troca de experiências, do compartilhamento de situações conflituosas e vivências em comum dos sujeitos do grupo, cada um pôde voltar para si mesmo e promover um encontro de sua vida.

Em seguida, foram distribuídas pelo chão, gravuras diversas, com pessoas em diversos momentos da vida, para que elas pudessem escolher uma e posteriormente falar acerca da escolha.

As figuras escolhidas representavam a projeção dos desejos almejados, refletindo os sonhos que muitas vezes são esquecidos, em conseqüência das dificuldades enfrentadas. As perspectivas de futuro e ascensão social dessas mulheres estavam muito relacionadas à existência de uma profissão, trabalhar e receber uma renda fixa.

Outras gravuras despertaram as atenções para medidas de segurança no ambiente de trabalho, gerando uma discussão acerca de estratégias de redução da exposição à violência.

Em relação à associação que se estabelece entre prostituição e violência, algumas prostitutas que estavam presentes já haviam sofrido ou presenciado algum tipo de agressão, tanto por parte de seus clientes como por outras meninas de programa, evidenciando-se, dessa forma, a insegurança e o risco a que estão expostas durante a realização de suas atividades profissionais.

A violência sexual cometida contra a mulher é de grande relevância por sua complexidade, sendo imprescindível a execução de ações de prevenção capazes de influenciar a realidade dessas mulheres ${ }^{(9)}$.

Percebeu-se, através das atitudes e reflexões das prostitutas do grupo, que a prostituição não é encarada como profissão, mas que tem um caráter de atividade temporária. Identificou-se insatisfação, desejo de mudar de vida, com esperança de que consigam ter uma melhor qualidade de vida e pleno desenvolvimento de suas cidadanias.

Tentou-se impor uma postura crítica e curiosa aos sujeitos da oficina, em face do objeto de seu conhecimento. Procurou-se o distanciamento de um processo de pura transferência de conhecimento, em que conhecer deixa de ser um ato criador e recriador para ser um "ato digestivo"(11). Através do processo reflexivo buscou-se que as prostitutas olhassem para o seu interior, questionando-se e conhecendo-se.

\section{REFLEXÕES FI NAIS}

As metodologias participativas são estratégias que permitem uma maior conscientização e influenciam positivamente nas mudanças de comportamento, induzindo a uma melhor condição de saúde. Dentre esses métodos, enfatiza-se o emprego de dinâmicas de grupo e oficinas em ambiente de sala de espera, que proporcionam um sentimento de valorização, eqüidade e inclusão do sujeito.

As oficinas utilizadas como técnicas de grupo em sala de espera para as prostitutas demonstraram ser atividades próprias para promover o processo de educação em saúde, a promoção da saúde e a prevenção de doenças, pois foi observado um processo de intensas trocas de experiências entre pessoas distintas e intercâmbios entre saberes, edificando, assim, o conhecimento entre as participantes destas oficinas.

Os enfermeiros como agentes promotores de saúde, devem buscar capacitação para o atendimento das necessidades psicossociais de pacientes e familiares a fim de melhorar sua atuação profissional.

A realização do presente relato poderá difundir ainda mais essa estratégia de educação em saúde, estimulando outros serviços e outros profissionais a transformar a realidade positivamente e a edificar um pensamento crítico de valorização do cliente. 


\section{REFERÊNCIAS}

1. Benzaken AS, Galbán Garcia E, Sardinha JCG, Pedrosa VL, Paiva V. Intervenção de base comunitária para a prevenção das DST/Aids na região amazônica, Brasil. Rev. Saúde Públ. 2007;41 Supl 2:118-26.

2. Andrade MCC. Mulheres prostituídas. Revista Videtur - Letras [Internet]. 2002 [cited 2008 mar 17];5:27-40. Available from: http://www. hottopos.com/seminario/sem2/cris1.htm. 3. Coordenação Nacional de DST/AIDS; Ministério da Saúde. Políticas e diretrizes de prevenção das DST/Aids entre mulheres. Brasília (Brasil): Ministério da Saúde; 2003.

4. Jornal de debates [Internet]. Rio de Janeiro: DiHITT - notícias (BR) [update 2007 mar 12, cited 2008 dec 31]. Turismo sexual no Ceará. Available from:

http://www.jornaldedebates.ig.com.br/debate/l ula-visita-bush-jogo-dos-americanos-

interessa/artigo/jogo-dos-americanos-nosinteressa-3.

5. Escola de Educação Física e Desportos [Internet]. Rio de Janeiro: UFRJ [update 2000 nov 25, cited 2005 ago 17]. Lazer e prostituição. Available from: http://www. lazer.eefd.ufri.br/antigos/lazer_pros tituicao_ll/prostituicao.htm.

6. Alves Filho M. Asas do desejo. Jornal da UNICAMP [Internet]. 2004 out 11 a 17 [cited 2008 dec 31]. Available from: http://www. unicamp.br/unicamp/unicamp_hoje /jornalPDF/ju269pag12.pdf

7. Aquino PS. Comportamento sexual de profissionais do sexo: risco para aquisição de DST? [monograph]. Fortaleza: Departamento de Enfermagem/UFC; 2005. 57p.

8. Schreiner L, Paim LL, Ramos F, Cunha Filho EV, Martins DM, Silva Junior CL, et al. Prevalência de sintomas depressivos em uma amostra de prostitutas de Porto Alegre. Rev. psiquiatr. Rio Gd. Sul. 2004;26(1): 13-20.

9. Cavalcanti LF, Gomes R, Minayo MCS. Representações sociais de profissionais de saúde sobre violência sexual contra a mulher: estudo em três maternidades públicas municipais do Rio de Janeiro, Brasil. Cad. Saúde Pública. 2006; 22(1):31-9.

10. Lei 8080, de 19 de setembro de 1990 Dispõe sobre as condições para a promoção, proteção e recuperação da saúde, a organização e 0 funcionamento dos serviços correspondentes, e dá outras providências. Diário Oficial da União (Brasília). 1990 Set 20.

11. Freire P. Ação cultural para a liberdade e outros escritos. Rio de Janeiro: Paz e Terra; 1982.

12. Ponte CMM, Fernandes VO, Gurgel MHC, Veras VS, Quidute ARP, Montenegro RM et al. Projeto sala de espera: uma proposta para a educação em diabetes. Revista Brasileira de Promoção da Saúde. 2006; 19(4): 197-202.

13. Instituto Nacional do Câncer; Ministério da Saúde. Câncer do colo do útero. Rio de Janeiro: Ministério da Saúde; 2007.

14. Maffacciolli R, Lopes MJM. Educação em saúde: a orientação alimentar através de atividades de grupo. Acta paul. enferm. 2005; 18(4): 439-45.

15. Teixeira ER, Veloso RC. O grupo em sala de espera: território de práticas e representações em saúde. Texto contexto-enferm. 2006; 15(2): 320-5.

16. Amaral MA, Pontes HA, Lopes LR, Massa TCSC, Fonseca RMGS. Oficinas de sexualidade: uma abordagem ampliada para se trabalhar com adolescentes. In: Anais do 8o Encontro de Extensão da UFMG; 2005 out 3-8; Belo Horizonte, Brasil. Belo Horizonte: UFMG; 2005.

17. Almeida NAM, Silva LA, Araújo NM. Conhecimento de acadêmicas de Enfermagem sobre disfunções sexuais femininas. Revista Eletrônica de Enfermagem [Internet]. 2005 [cited 2007 abr 4]; 7(2): 138-47. Available from: http://www.fen.ufg.br/revista/revista7_2/origin al_01.htm.

18. Tornis NHM, Lino AIA, Santos MAM, Lopes CLR, Barbosa MA, Siqueira KM. Sexualidade e anticoncepção: o conhecimento do escolar/adolescente. Rev. Eletr. Enf. [Internet]. 2005 [cited 2008 dec 31];7(3):344-50. Available from: http://www.fen.ufg.br/Revista/revista7 3/origin al_12.htm.

19. Guimarães K, Merchán-Hamann E. Comercializando fantasias: a representação social da prostituição, dilemas da profissão e a construção da cidadania. Rev. Estud. Fem. 2005; 13(3): 525-44.

20. Andraus LMS, Oliveira LMAC, Minamisava R, 
Moraes MLC, Costa PB, Aquino OS, Pinheiro AKB. Educação em saúde com prostitutas de Fortaleza: relato de experiência. Rev. Eletr. Enf. [Internet]. 2008;10(4):1144-51. Available from: http://www. fen.ufg. br/revista/v10/n4/v10n4a27.htm.

Munari DB, Borges IK. Ensinando e aprendendo: uma experiência com grupos de pais de crianças hospitalizadas. Revista Eletrônica de Enfermagem [internet]. 2004 [cited 2008 dec 31];6(1):98-103. Available from: http://www.fen.ufg.br/revista/revista6_1/r2_pais. html.

Artigo recebido em 30.09.07.

Aprovado para publicação em 31.12.08. 